\title{
The Most Common Clinical Features of Kawasaki Disease Patients in King Abdulaziz Medical City
}

Khalid Talal Alghamdi ${ }^{1}$, Rahaf Abdulmalik Waggass ${ }^{2}$, Syed Sameer Aga ${ }^{3}$, Abdulaziz Ahmed Alrohaili ${ }^{1}$, Ali Hassan Alaidroos ${ }^{1}$, Mohammed Dakhilallah Alghamdi ${ }^{1}$, Mohannd Khalid Algamdi ${ }^{1}$, Ashwaq Talal Alghamdi 4

1. College of Medicine, King Saud bin Abdulaziz University for Health Sciences, King Abdullah International Medical Research Centre, King Abdulaziz Medical City, National Guard Health Affairs, Jeddah, SAU 2. Cardiology, King Saud bin Abdulaziz University for Health Sciences, Jeddah, SAU 3. Basic Medical Sciences, College of Medicine, King Saud bin Abdulaziz University for Health Sciences, King Abdullah International Medical Research Centre, King Abdulaziz Medical City, National Guard Health Affairs, Jeddah, SAU 4. Cardiology, King Abdulaziz University Faculty of Medicine, Jeddah, SAU

Corresponding author: Khalid Talal Alghamdi, alghamdi075@ksau-hs.edu.sa

\section{Abstract \\ Background}

Kawasaki disease (KD) is an acute idiopathic vasculitis affecting the small and medium-sized arteries especially coronary artery (CA). it occurs in childhood mostly below five years of age.

\section{Objective}

This study aims to identify the most common clinical features among KD patients in King Abdulaziz Medical City in Jeddah, Saudi Arabia from 1/1/1982 to 31/12/2018.

\section{Methods}

A case series study was conducted in all KD patients that were admitted to the King Abdulaziz Medical City from 1/1/1982 to 31/12/2018 except those who were diagnosed in other hospitals or whose diagnosis has later changed. The identification of patient was done by using the International Classification of Diseases (ICD) coding for KD (ICD9 446.1). Our data consisted of the patient's file number, age at presentation, gender, whether the patients received IVIG treatment or not, number of days of fever before starting IVIG treatment, response to IVIG treatment, season in which the symptoms started and clinical features based on body's system.

\section{Result}

The study included 18 patients, 11 males showed that (55.6\%) of patients met the criteria of typical KD and most of them were less than five years old. In addition, most patients were reported to have polymorphous rash, cough, irritability, vomiting, and a murmur. All patients who received intravenous immunoglobulin (IVIG) treatment which demonstrated an improvement even though those who started the treatment after 10 days of fever.

\section{Conclusion}

In typical KD patients, the distribution of the clinical features was almost identical. However, there were some variations in them among atypical KD patients. Moreover, Evan though KD in our region is not common as in Japan, the incidence of giant aneurism was higher. In addition to that, this study and other study conducted in Saudi Arabia found that screened patients reported tachycardia more than patients in Japan.

\section{Recommendation}

As $\mathrm{KD}$ is still idiopathic, we recommend more details to be collected from the patients, especially consanguinity as it is common in Saudi Arabia

Categories: Cardiac/Thoracic/Vascular Surgery, Cardiology, Pediatrics

Keywords: kawasaki disease (kd), vascular inflammation, vasculitis, king abdulaziz medical city, saudi arabia

\section{Introduction}

Kawasaki disease (KD) is an acute idiopathic vasculitis occurs mainly in children under five years of age, affecting small- and medium-sized arteries, especially coronary arteries. KD was initially reported by Tomisakau Kawasaki in 1967 in Japan; and thereafter, KD was recognized all over the world [1,2]. KD is 
characterized by sentinel features of fever that lasts for at least five days, cervical lymphadenopathy, bilateral conjunctivitis, oral mucosal changes, polymorphous rash, and swelling or redness of extremities $[3,4]$. Accordingly, $\mathrm{KD}$ is classified into two groups: (A) complete (typical) $\mathrm{KD}$, which is defined by the presence of at least four of the five symptoms in addition to the fever [1]; and (B) incomplete (atypical) KD, defined by appearance of at least two of the five symptoms in addition to the fever [1]. Coronary arterial lesions are the most important complication of KD that may eventually lead to myocardial infarction [5]. KD is associated with other conditions such as mild hepatitis, aseptic meningitis, and pericardial effusion [3]. Intravenous immunoglobulin (IVIG) and aspirin are the treatment regimen used in KD to decrease the percentage of developing coronary arterial lesions [1].

Numerous studies around the world have reported similar clinicopathological characteristics of KD [6,7]. KD has been found to be more prevalent in Asian countries, especially in Japan, with an annual incidence of 308 per 100,000 children of less than five years of age, while in Europe incidence ranges between 4.9 and 15.2 per 100,000 children of less than five years of age [8]. Locally in Saudi Arabia, children of five years old incidence were reported as 7.4 per 100,000 [1].

Since there is limited data available for KD in Saudi Arabia, we carried out this case series to identify the clinical characteristics of patients affected by KD in King Abdulaziz Medical City in Jeddah, Saudi Arabia and to compare them with other local and international published data.

\section{Materials And Methods}

This case series study was undertaken in the Pediatrics Department of King Abdulaziz Medical City, National Guard Health Affairs in Jeddah, Saudi Arabia. The study was approved by the Institutional Review Board (IRB) of the hospital. The identification of the KD was confirmed by using the International Classification of Diseases (ICD) coding for KD (ICD9 446.1) retrospectively on all the children that were admitted to the Hospital from 1/1/1982 to 31/12/2018. Individual patient data were reviewed to confirm the diagnosis of KD according to the American Academy of Pediatrics and the American Heart Association (AHA) guidelines established in 2004. The patient's demographic, clinical, laboratory and echocardiographic data were recorded.

We collected the data by using patients' old system files. Our data consisted of the patient's file number, age at presentation, gender, whether the patients received IVIG treatment or not, number of days of fever before starting IVIG treatment, response to IVIG treatment, season in which the symptoms started and clinical features based on body's system. All patients less than 15 years old who were diagnosed with KD were included in this study, except those patients who were diagnosed in other hospitals or whose diagnosis has later changed. Coding sheets were used to protect the identities of patients. Each patient was given a code linked to his/her medical record number (MRN) to preserve the patient's information.

\section{Statistical analysis}

Excel was used for data entry, and Statistical Package for the Social Sciences (SPSS) version 23 (IBM Corp., Armonk, NY) was used for calculating the mean and standard deviation for quantitative data, frequency and percentage for qualitative data.

\section{Results}

The study included 18 patients, 11 of them were males. The male-to-female ratio was 1.6:1. Ten patients (55.5\%) met the criteria of typical KD while the rest were diagnosed as atypical KD. Moreover, 14 patients ( $77.7 \%$ ) found to be five years old or less while the rest were above the age of five years. The mean age was 52 months for males and 43 months for females.

All patients who received IVIG treatment showed improvement regardless of the number of days of fever before starting the treatment. However, there were two male patients who did not receive IVIG treatment (Table 1). 


\section{Cureus}

\begin{tabular}{|c|c|c|c|c|c|c|}
\hline Gender (No.) & \multicolumn{3}{|l|}{ Male (11) } & \multicolumn{3}{|l|}{ Female (7) } \\
\hline \multirow{2}{*}{ Management } & \multicolumn{2}{|c|}{ Received IVIG treatment } & Did not receive IVIG & \multicolumn{2}{|c|}{ Received IVIG treatment } & Did not receive IVIG \\
\hline & \multicolumn{2}{|l|}{9} & 2 & \multicolumn{2}{|l|}{7} & 0 \\
\hline \multirow{2}{*}{$\begin{array}{l}\text { Number of days of fever before } \\
\text { starting IVIG treatment }\end{array}$} & $\begin{array}{l}\text { Less than } \\
10 \text { days }\end{array}$ & $\begin{array}{l}\text { More than } \\
10 \text { days }\end{array}$ & - & $\begin{array}{l}\text { Less than } \\
10 \text { days }\end{array}$ & $\begin{array}{l}\text { More than } \\
10 \text { days }\end{array}$ & - \\
\hline & 6 & 3 & - & 4 & 3 & - \\
\hline
\end{tabular}

\section{TABLE 1: IVIG treatment.}

IVIG: intravenous immunoglobulin.

As demonstrated in Table 2, the most common respiratory symptom related to KD was cough, and there were two patients reported to have upper respiratory tract infection. Bronchial asthma was found in two patients and nine patients were reported to have cardiovascular symptoms. The most common sign was a murmur and a giant aneurysm was reported in one patient. Cardiovascular symptoms were absent in nine patients which is $50 \%$ of the total number of patients. Regarding the musculoskeletal system, two patients were suffering from arthralgia and one of the patients reported other symptoms like arthritis, skeletal dysplasia, and kyphoscoliosis. Moreover, irritability was the commonest symptom of the nervous system with one of the patients was reported with torticollis. Eleven patients were having gastrointestinal symptoms of which the most common was vomiting found in 6 patients. Other gastrointestinal symptoms including abdominal pain and diarrhea, both in four patients. For the seasonal distribution of incidence, the number of cases in summer was 6 (33.3\%), which was the highest, followed by spring and autumn with 5 (27.7\%) patients for each, lastly the winter with 2 (11.1\%) patients. There were no deaths reported.

\begin{tabular}{|c|c|c|c|c|}
\hline \multirow{2}{*}{$\begin{array}{l}\text { Gender (No.) } \\
\text { Clinical features }\end{array}$} & \multicolumn{2}{|l|}{ Male (11) } & \multicolumn{2}{|c|}{ Female (7) } \\
\hline & Present & Absent & Present & Absent \\
\hline \multicolumn{5}{|l|}{ Classical criteria } \\
\hline Acute lymphadenopathy & $7(64 \%)$ & $4(36 \%)$ & $4(57 \%)$ & $3(43 \%)$ \\
\hline Conjunctival injection & $7(64 \%)$ & $4(36 \%)$ & $3(43 \%)$ & $4(57 \%)$ \\
\hline Polymorphous rash & $8(73 \%)$ & $3(27 \%)$ & $7(100 \%)$ & $0(0 \%)$ \\
\hline Lips and oral mucosal changes & $6(55 \%)$ & $5(45 \%)$ & $6(86 \%)$ & $1(14 \%)$ \\
\hline Change in extremities (Swelling or erythema) & $8(73 \%)$ & $3(27 \%)$ & $6(86 \%)$ & $1(14 \%)$ \\
\hline \multicolumn{5}{|l|}{ Other features } \\
\hline Respiratory symptoms (Cough, rhinorrhea, throat congestion)* & $5(45 \%)$ & $6(55 \%)$ & $3(43 \%)$ & $4(57 \%)$ \\
\hline Cardiovascular symptoms (Murmur, tachycardia)* & $5(45 \%)$ & $6(55 \%)$ & $4(57 \%)$ & $3(43 \%)$ \\
\hline Musculoskeletal symptoms (Arthritis, arthralgia) ${ }^{\star}$ & $3(27 \%)$ & $8(73 \%)$ & $0(0 \%)$ & $7(100 \%)$ \\
\hline Nervous system symptoms (Irritability, lethargy) ${ }^{\star}$ & $2(18 \%)$ & $9(82 \%)$ & $3(43 \%)$ & $4(57 \%)$ \\
\hline Gastrointestinal symptoms (Vomiting, decreased oral intake) ${ }^{\star}$ & $8(73 \%)$ & $3(27 \%)$ & $3(43 \%)$ & $4(57 \%)$ \\
\hline Dermatological symptoms (Rash, skin peeling) ${ }^{\star}$ & $10(91 \%)$ & $1(9 \%)$ & $6(86 \%)$ & $1(14 \%)$ \\
\hline Hematological symptoms (Leukocytosis, thrombocytosis) & $3(27 \%)$ & $8(73 \%)$ & $1(\% 17)$ & $6(\% 83)$ \\
\hline
\end{tabular}

\section{TABLE 2: Clinical features of the patients.}

${ }^{*}$ These are the most common symptoms. 


\section{Discussion}

King Abdulaziz Medical City is one of the largest hospitals in Jeddah. Among the 18 patients, 10 (55.6\%) of them were typical for KD and 8 (44.4\%) presenting with atypical KDs. The number of Our patients was small but close to other studies in the region. [1,7] The distribution of symptoms was almost identical in typical $\mathrm{KD}$ patients. However, in atypical KD patients, the most common symptom was polymorphous rash while it was not reported as the commonest feature of KD in other researches [5,7]. Moreover, the least reported symptom was conjunctival injection. However, studies in east Asia show that the conjunctival injection was the most common reported classical symptom [9,10]. In contrast to some of the European and most Asian studies where the maximum incidence occurred in winter, in our patients the highest incidence was in summer and the least in winter $[11,12]$.

After dermatological changes which are included in the classical features of KD, gastrointestinal symptoms were the most reported finding in this study and also in another local study [7]. Vomiting was the highest noted symptom in our research, much like that found in Japan study as well as locally in Saudi Arabia $[6,7,8]$. Decreased oral intake was reported in $27.7 \%$ (five patients) compared to $37.5 \%$ in a local research study [7]. In addition, some research studies reported organomegaly. Only one of our patients was reported with splenomegaly $[3,7]$. As opposed to a research study that has been done in Spain, irritability was the least common symptom while being the highest in the research findings of Spain [8]. In our research, Even though we have only 18 patients one of them reported with giant aneurysms, which represent $(5.6 \%)$. While in Japan, only (0.24\%) had giant aneurysms [13]. Moreover, our patient was female in contrast to other research that showed that males have higher occurrence of giant aneurysms [8]. In our study, the incidence of tachycardia was $11.11 \%$, which was $49 \%$ in another local study [1]. On the other hand, the incidence in japan was $0.07 \%$. This suggests that Saudi Arabia has a higher incidence of tachycardia in this disease.

Except for two patients, all other patients received IVIG treatment, and regardless of the duration of fever before receiving IVIG treatment, all cases demonstrated an improvement; however, it is not suggested to delay the treatment with IVIG because most research studies have shown a better response rate if started prior to 10 days of fever $[7,8,14]$. No deaths were reported in our study. Most other studies even with large number of cases have also reported zero or a low death rate $[8,9,13]$.

\section{Conclusions}

In conclusion, since $\mathrm{KD}$ is rare in our region, physicians should have a high index of suspicion for this diagnosis. The etiology and pathogenesis of KD are not fully established, so we recommend better reporting of symptoms and clinical findings in patients with $\mathrm{KD}$, particularly because it is common. Being a case series study in one center was a limitation in our study and it is recommended to perform a prospective multicenter study in the future to obtain added information pertaining to our nation.

\section{Additional Information \\ Disclosures}

Human subjects: Consent was obtained or waived by all participants in this study. King Abdullah International Medical Research Centre issued approval SP19/201/J. Animal subjects: All authors have confirmed that this study did not involve animal subjects or tissue. Conflicts of interest: In compliance with the ICMJE uniform disclosure form, all authors declare the following: Payment/services info: All authors have declared that no financial support was received from any organization for the submitted work. Financial relationships: All authors have declared that they have no financial relationships at present or within the previous three years with any organizations that might have an interest in the submitted work. Other relationships: All authors have declared that there are no other relationships or activities that could appear to have influenced the submitted work.

\section{References}

1. Lardhi AA: Kawasaki disease: a university hospital experience. Saudi J Med Med Sci. 2013, 1:35-9. 10.4103/1658-631X.112923

2. Scuccimarri R: Kawasaki disease. Pediatr Clin North Am. 2012, 59:425-45. 10.1016/j.pcl.2012.03.009

3. Shamsizadeh A, Ziaei Kajbaf T, Razavi M, Cheraghian B: Clinical and epidemiological characteristics of kawasaki disease. Jundishapur J Microbiol. 2014, 7 :e11014. 10.5812/jjm.11014

4. Freeman AF, Shulman ST: Kawasaki disease: summary of the American Heart Association Guidelines. Am Fam Physician. 2006, 74:1141-8.

5. Bhatnagar SK, Paul G, Subramanian R, Al Hosni MS, Al Khusaiby SM: Kawasaki disease in Oman--a clinical study. J Trop Pediatr. 2003, 49:361-6. 10.1093/tropej/49.6.361

6. Nakamura Y, Yashiro M, Uehara R, et al.: Epidemiologic features of Kawasaki disease in Japan: results of the 2009-2010 nationwide survey. J Epidemiol. 2012, 22:216-21. 10.2188/jea.je20110126

7. Al-Harbi KM: Kawasaki disease in Western Saudi Arabia . Saudi Med J. 2010, 31:1217-20.

8. Fernandez-Cooke E, Barrios Tascón A, Sánchez-Manubens J, et al.: Epidemiological and clinical features of Kawasaki disease in Spain over 5 years and risk factors for aneurysm development. (2011-2016): KAWARACE study group. PLoS One. 2019, 14:e0215665. 10.1371/journal.pone.0215665

9. Xie LP, Yan WL, Huang M, Huang MR, Chen S, Huang GY, Liu F: Epidemiologic features of Kawasaki disease 


\section{Cureus}

in Shanghai from 2013 through 2017. J Epidemiol. 2020, 30:429-35. 10.2188/jea.JE20190065

10. Kim GB, Park S, Eun LY, et al.: Epidemiology and clinical features of Kawasaki disease in South Korea, 20122014. Pediatr Infect Dis J. 2017, 36:482-5. 10.1097/INF.0000000000001474

11. Burns JC, Cayan DR, Tong G, et al.: Seasonality and temporal clustering of Kawasaki syndrome. Epidemiology. 2005, 16:220-5. 10.1097/01.ede.0000152901.06689.d4

12. Jakob A, Whelan J, Kordecki M, et al.: Kawasaki disease in Germany: a prospective, population-based study adjusted for underreporting. Pediatr Infect Dis J. 2016, 35:129-34. 10.1097/INF.0000000000000953

13. Mauro A, Fabi M, Da Frè M, et al.: Kawasaki disease: an epidemiological study in central Italy. Pediatr Rheumatol Online J. 2016, 14:22. 10.1186/s12969-016-0084-6

14. Agarwal S, Agrawal DK: Kawasaki disease: etiopathogenesis and novel treatment strategies . Expert Rev Clin Immunol. 2017, 13:247-58. 10.1080/1744666X.2017.1232165 